\title{
Joint Beamforming Optimization for the Intelligent Reflecting Surface Assisted Wireless Surveillance System
}

\author{
Yan Pan, Zhixiang Deng* \\ College of Internet of Things Engineering, Hohai University, Changzhou, China \\ Email address: \\ y1506205850@163.com (Yan Pan),dengzhixiang@gmail.com (Zhixiang Deng) \\ ${ }^{*}$ Corresponding author
}

To cite this article:

Yan Pan, Zhixiang Deng. Joint Beamforming Optimization for the Intelligent Reflecting Surface Assisted Wireless Surveillance System. Mathematics and Computer Science. Vol. 6, No. 1, 2021, pp. 1-7. doi: 10.11648/j.mcs.20210601.11

Received: December 27, 2020; Accepted: January 7, 2021; Published: January 18, 2021

\begin{abstract}
The intelligent reflecting surface (IRS), which consists of a large number of reflecting units, can adjust the phase shifts of its reflecting units to strengthen the desired signal and/or suppress the undesired signal. In this paper, we consider an IRS-assisted wireless surveillance system where an IRS is deployed to assist the legal surveillance receiver E to monitor the information transmission of the suspicious link from AP to the suspicious receiver B. Two communication scenarios assuming whether the suspicious link is aware of the existence of the monitor are considered. The optimization problem under the constraint that the achievable rate at the monitor $\mathrm{E}$ is larger than that at the suspicious receiver $\mathrm{B}$ is proposed to jointly optimize the beamforming vector at the AP and the phase shift matrix at the IRS to maximize the achievable eavesdropping rate. To solve this non-convex problem, we introduce the semi-definite relaxation (SDR) approach and the alternating optimization (AO) method to convert the non-convex optimization problem to a series of semi-definite programs (SDPs) and solve the SDPs iteratively. Simulation results show that the assistance of IRS can greatly improve the performance of the surveillance, and achieves significant advantages over the traditional relay-assisted wireless surveillance system.
\end{abstract}

Keywords: Intelligent Reflecting Surface (IRS), Wireless Surveillance System, Semi-definite Relaxation (SDR), Alternating Optimization (AO)

\section{Introduction}

Recently, the fifth-generation (5G) wireless communication networks have been applied in commercial deployment [1]. It aims at realizing ultra-high spectrum and energy efficiency, ultra-dense user connectivity, and very low latency. However, due to the open nature of the wireless medium, the security problem presents a great threat to the information transmission in the wireless communication systems. In addition, the ubiquitous accessibility of wireless communications is also vulnerable to malicious exploitation. The legal surveillance of suspicious information transmission is a very urgent need. The achievable rate of the monitor in the surveillance system should be higher than that of the suspicious receiver such that the information from the suspicious transmitter could be decoded successfully at the monitor [2].

In the wireless communication systems, technologies such as the relaying, large-scale MIMO, artificial noise, are adopted to improve the security of the wireless communication systems [3-5]. On the other hand, in the wireless surveillance system, the monitor is a legitimate eavesdropper that tries to decode the information of the suspected communication link [6].

To improve the secrecy rate of wireless communication systems, the MIMO [7] and relaying [8] technologies are often used to improve the performance of the wireless communication systems. However, a large number of RF chains should be used in the MIMO systems. It leads to excessive hardware consumption. The introduction of active relays in the relaying systems leads to more energy consumption. The emergence of IRS technology has successfully alleviated these problems [9]. The MIMO and relaying technologies which are used to assist wireless communication, are active communication devices. The IRS is passive and has the advantages of lower energy consumption, better flexibility, and higher efficiency. The IRS can be programmed to respond to external stimuli. Their static interaction can be changed, meaning that their interaction with 
the crashing wireless waves can be manipulated.

The achievable rate can be improved by jointly optimizing the active beamforming and passive beamforming in the IRS-assisted systems $[10,11]$. The beamforming vector of the transmitter and the reflecting matrix are jointly optimized to maximize the achievable secrecy rate [12]. It was demonstrated that deploying large IRS reflectors is more effective than enlarging the antenna numbers of the transmitters [13]. Jamming is not only working well with the relay [14], but also plays a vital role in the IRS-assisted wireless system [15].

Through the so-called spoofing relay attack in the relay-assisted surveillance system, eavesdroppers can launch spoofing relay attacks, which significantly increases the rate of information leakage from the suspicious source [16]. The relay acts as a power splitting receiver. It divides the received signal into two parts for information interception and spoofing relay, respectively. Three possible spoofing relay schemes according to the eavesdropping conditions were discussed [17].

In this paper, we design an IRS-assisted surveillance system by jointly optimizing the active beamforming of AP and the passive beamforming of IRS to improve the surveillance system performance. Two scenarios are considered assuming whether the suspicious AP and the receiver B are aware of the presence of the monitor $\mathrm{E}$. We abuse monitor-unaware and monitor-aware to denote the two cases, respectively. We jointly optimize the active beamforming at the AP and the passive beamforming by the phase shifters at the IRS to maximize the achievable rate at the user under the constraint that the achievable rate at the monitor is larger than that at the receiver in the monitor-aware case. And in the monitor-unaware case, we aim to optimize the passive beamforming only. The optimization problems in both cases are non-convex and are hard to be solved directly. To solve the non-convex optimization problems, we introduce the semi-definite relaxation (SDR) method and alternating optimization (AO) approach to convert the original optimization problem to a series of semi-definite programs (SDPs) which are solved iteratively. Numerical results are then presented to show the achievable rate performance of the IRS-assisted surveillance system.

Notations: Scalars, vectors and matrices are denoted by italic letters, bold-face lower-case letters and bold-face upper-case letters respectively. The Euclidean norm of a complex-valued vector $\mathbf{x}$ is denoted by $\|\mathbf{x}\|$. The space of $m \times n$ complex-valued matrices is denoted by $C^{m \times n}$. The notation $\operatorname{diag}(\mathbf{x})$ denotes a diagonal matrix with each diagonal element being the corresponding element in $\mathrm{x}$ and $\arg (\mathbf{x})$ is a vector each element of which is the phase of the corresponding element in $\mathrm{x} . \operatorname{Tr}(\mathrm{A})$ and $\mathbf{A}^{-1}$ means the trace and inverse of a square matrix $\mathbf{A} . \mathbf{A} \geqslant 0$ means the matrix $\mathbf{A}$ is positive semi-definite. The conjugate, transpose and rank of any matrix $\mathbf{A}$ are denoted by $\mathbf{A}, \mathbf{A}^{H}, \operatorname{rank}(\mathrm{A})$ respectively.

\section{System Model and Problem Formulation}

\subsection{System Model}

We consider an IRS-assisted wireless surveillance system, as shown in Figure 1, where a legitimate monitor E aims at monitoring a MISO suspicious communication link from the AP to the suspicious receiver B with the assistance of the IRS. The legitimate monitor $\mathrm{E}$ and suspicious receiver $\mathrm{B}$ are equipped with single antenna while the transmitter AP equipped with $M$ antennas. The IRS is assumed to be composed by $N$ passive reflecting units, denoted by the set $\mathcal{N}=\{1, \ldots, N\}$, which is connected to a controller. Due to significant path loss, it is assumed that the power of the signals that are reflected by the IRS two or more times is negligible and thus ignored. We consider a quasi-static block fading channel model for all channels involved and focus on one particular channel that remains approximately constant. We assume that perfect CSI of all channels is available at the AP and the monitor $\mathrm{E}$ via channel training and feedback from the IRS/user.

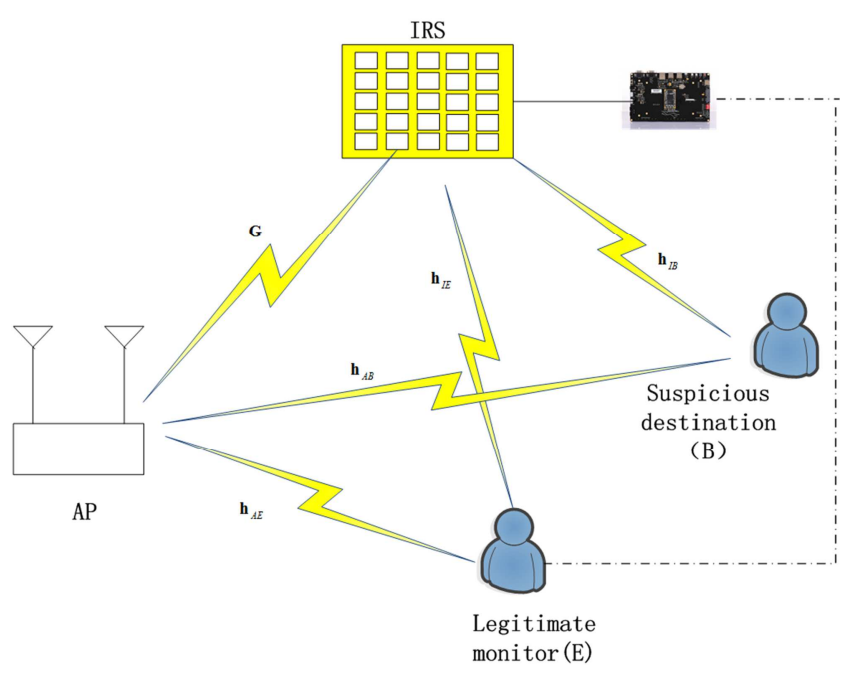

Figure 1. An IRS-assisted surveillance system.

The baseband equivalent channels from AP to IRS, $\mathrm{B}$ and $\mathrm{E}$ are denoted by $\mathrm{G} \in C^{M \times N}, \quad h_{\mathrm{AB}} \in C^{M \times 1}, h_{\mathrm{AE}} \in$ $C^{M \times 1}$ respectively, and the channels from IRS to $\mathrm{B}$ and $\mathrm{E}$ denoted by $h_{\mathrm{IB}} \in C^{N \times 1}, \quad h_{\mathrm{IE}} \in C^{N \times 1}$. Let $\boldsymbol{\Phi}=\operatorname{diag}\left(\beta e^{j \theta_{1}}, \ldots, \beta e^{j \theta_{n}}, \ldots \beta e^{j \theta_{N}}\right)$ denote the diagonal phase-shift matrix for the IRS, where $\theta_{n} \in[0,2 \pi]$ and $\beta \in[0,1]$ are the phase shift and amplitude reflection coefficient on the combined incident signal of the $n$-th element respectively. The transmitted signal from AP is $\mathbf{x}=\mathbf{W} S$, where $\mathrm{w} \in C^{M \times 1}$ is the information beamforming vector, $\|\mathbf{w}\|^{2} \leq P, P$ is the maximum transmit power at AP, $s \sim \mathcal{C N}(0,1)$ is a circularly symmetric complex Gaussian (CSCG) random variable denoting the information carrying signal.

The information received at $\mathrm{B}$ and $\mathrm{E}$ can be expressed as: 


$$
\begin{aligned}
& y_{B}=\left(\mathbf{h}_{I B}^{H} \mathbf{\Phi} \mathbf{G}+\mathbf{h}_{A B}^{H}\right) \mathbf{x}+n_{B} \\
& y_{E}=\left(\mathbf{h}_{I E}^{H} \mathbf{\Phi} \mathbf{G}+\mathbf{h}_{A E}^{H}\right) \mathbf{x}+n_{E}
\end{aligned}
$$

where $n_{B}$ and $n_{E}$ are the independent and identically distributed (i.i.d.) Gaussian random noises at the B and E with zero mean and variances $\sigma_{B}^{2}$ and $\sigma_{E}^{2}$ respectively. The achievable rate at the suspicious receiver $\mathrm{B}$ and the monitor $\mathrm{E}$ is given by

$$
\begin{aligned}
& R_{B}=\log _{2}\left(1+\frac{\left|\left(\mathbf{h}_{I B}^{H} \mathbf{\Phi} \mathbf{G}+\mathbf{h}_{A B}^{H}\right) \mathbf{w}\right|^{2}}{\sigma_{B}^{2}}\right) \\
& R_{E}=\log _{2}\left(1+\frac{\left|\left(\mathbf{h}_{I E}^{H} \mathbf{\Phi} \mathbf{G}+\mathbf{h}_{A E}^{H}\right) \mathbf{w}\right|^{2}}{\sigma_{E}^{2}}\right)
\end{aligned}
$$

\subsection{Problem Formulation}

In this paper, we consider two scenarios assuming whether the suspicious AP and receiver B are aware of the presence of the monitor E. In the first case, we assume neither AP nor B is aware of the presence of $\mathrm{E}$. In the second case, AP is aware of the presence of $\mathrm{E}$ and is willing to cooperate with $\mathrm{E}$ under the surveillance of $E$ to improve its information rate. We abuse monitor-unaware and monitor-aware to denote the two cases respectively. In both cases, it is assumed that no physical-layer security coding is applied by the AP to prevent the eavesdropping by $\mathrm{E}$.

For the monitor-unaware case, the AP transmit information to $B$ with maximum ratio transmission (MRT), i.e. the information beamforming vector $\mathbf{W}$ is set to be $\mathbf{w}=\sqrt{P} \frac{\mathbf{h}_{A B}}{\left\|\mathbf{h}_{A B}\right\|}$. The monitor $\mathrm{E}$ tries to adjust the reflecting coefficients of the IRS to maximize the achievable rate of the AP $R_{B}$ under the constraint $R_{E} \geq R_{B}$ such that more information can be surveilled by $\mathrm{E}$. The optimization problem is expressed as

$$
\begin{aligned}
(\mathbf{P 1}): \max _{\boldsymbol{\Phi}} & R_{E} \\
\text { s.t. } & R_{B} \leq R_{E} \\
& 0 \leq \theta_{n}<2 \pi, \forall n=1,2, \ldots, N
\end{aligned}
$$

For the monitor-aware case, the AP is aware of the presence of the monitor $\mathrm{E}$ and is willing to cooperate with the monitor to improve its own rate. Therefore, the active beamforming vector $\mathbf{W}$ and the reflecting coefficient matrix $\boldsymbol{\Phi}$ can be jointly optimized to maximize the information rate under the constraint that the monitor can decode the information of the AP successfully, i.e. $R_{E} \geq R_{B}$. The optimization problem can be expressed as

$$
\begin{aligned}
& \text { (P2): } \max _{\mathbf{w}, \boldsymbol{\Phi}} R_{B} \\
& \text { s.t. } \quad R_{B} \leq R_{E} \\
& \|\mathbf{w}\|^{2} \leq P \\
& 0 \leq \theta_{n}<2 \pi, \forall n=1,2, \ldots, N
\end{aligned}
$$

where $\mathrm{P}$ is the average transmit power at the AP.

\section{Solution to the Optimization Problems}

As can be seen, (P1) is a special case of (P2) by fixing $\mathbf{w}=\sqrt{P} \frac{\mathbf{h}_{A B}}{\left\|\mathbf{h}_{A B}\right\|}$. Thus, we mainly focus on the solution to the problem (P2). The problem ( $\mathrm{P} 2)$ is a non-convex problem since the beamforming vectors are in quadratic terms. It's hard to solve this optimization problem directly. So, we deploy an SDR-based algorithm to convert the original optimization problems to SDPs by optimizing the phase shift matrix and the beamforming vector iteratively with the other fixed.

The problem $(\mathrm{P} 2)$ is equivalently written as

$$
\begin{aligned}
& \text { (P3): } \max _{\mathbf{w}, \mathbf{\Phi}}\left|\left(\mathbf{h}_{I B}^{H} \mathbf{\Phi} \mathbf{G}+\mathbf{h}_{A B}^{H}\right) \mathbf{w}\right|^{2} \\
& \text { s.t. }\left|\left(\mathbf{h}_{I E}^{H} \mathbf{\Phi} \mathbf{G}+\mathbf{h}_{A E}^{H}\right) \mathbf{w}\right|^{2} \geq\left|\left(\mathbf{h}_{I B}^{H} \mathbf{\Phi} \mathbf{G}+\mathbf{h}_{A B}^{H}\right) \mathbf{w}\right|^{2} \\
& \|\mathbf{w}\|^{2} \leq P \\
& 0 \leq \theta_{n}<2 \pi, \forall n=1,2, \ldots, N
\end{aligned}
$$

For any given $\mathbf{W}$, the optimization problem goes to

$$
\begin{array}{cl}
\text { (P4): } \max _{\mathbf{\Phi}} & \left|\left(\mathbf{h}_{I B}^{H} \mathbf{\Phi} \mathbf{G}+\mathbf{h}_{A B}^{H}\right) \mathbf{w}\right|^{2} \\
\text { s.t. } & \left|\left(\mathbf{h}_{I E}^{H} \mathbf{\Phi G}+\mathbf{h}_{A E}^{H}\right) \mathbf{w}\right|^{2} \geq\left|\left(\mathbf{h}_{I B}^{H} \mathbf{\Phi G}+\mathbf{h}_{A B}^{H}\right) \mathbf{w}\right|^{2} \\
& 0 \leq \theta_{n}<2 \pi, \forall n=1,2, \ldots, N
\end{array}
$$

Define $\quad \mathbf{h}_{b}=\operatorname{diag}\left(\mathbf{h}_{I B}^{H}\right) \mathbf{G w} \quad, \quad \mathbf{h}_{e}=\operatorname{diag}\left(\mathbf{h}_{I E}^{H}\right) \mathbf{G w} \quad, \quad$ let $\mathbf{v}=\left[v_{1}, \ldots, v_{N}\right]^{H}, v_{n}=e^{j \theta_{n}}, \forall n$. The constraints become to $\left|v_{n}\right|=1, \forall n=1, \ldots N \quad, \quad$ define $\quad \mathbf{h}_{I B}^{H} \mathbf{\Phi} \mathbf{G w}=\mathbf{v}^{H} \mathbf{h}_{b} \quad$, $\mathbf{h}_{I E}^{H} \mathbf{\Phi} \mathbf{G w}=\mathbf{v}^{H} \mathbf{h}_{\mathrm{e}}, h_{B w}=\mathbf{h}_{A B}^{H} \mathbf{w}, h_{E w}=\mathbf{h}_{A E}^{H} \mathbf{w}$, problem (P4) is rewritten as

$$
\begin{aligned}
\text { (P5): } \max _{\mathbf{v}} & \left|\mathbf{v}^{H} \mathbf{h}_{b}+h_{B w}\right|^{2} \\
\text { s.t. } & \left|\mathbf{v}^{H} \mathbf{h}_{e}+h_{E w}\right|^{2} \geq\left|\mathbf{v}^{H} \mathbf{h}_{b}+h_{B w}\right|^{2} \\
& \left|v_{n}\right|=1, \forall n=1, \ldots, N
\end{aligned}
$$

Problem (P5) is a non-convex problem. Define $\mathbf{Q}_{B}=\left[\begin{array}{cc}\mathbf{h}_{b} \mathbf{h}_{b}^{H} & \mathbf{h}_{b} h_{B w}^{H} \\ h_{B w} \mathbf{h}_{b} & 0\end{array}\right], \quad \mathbf{Q}_{E}=\left[\begin{array}{cc}\mathbf{h}_{e} \mathbf{h}_{e}^{H} & \mathbf{h}_{e} h_{E w}^{H} \\ h_{E w} \mathbf{h}_{e} & 0\end{array}\right], \quad \overline{\mathbf{v}}=\left[\begin{array}{c}\mathbf{v} \\ t\end{array}\right]$, introduce $t$ as an auxiliary variable, problem (P5) can be equivalently written as

$$
\text { (P6): } \begin{aligned}
\max _{\overline{\mathbf{v}}} & \overline{\mathbf{v}}^{H} \mathbf{Q}_{B} \overline{\mathbf{v}}+\left|h_{B w}\right|^{2} \\
\text { s.t. } & \overline{\mathbf{v}}^{H} \mathbf{Q}_{B} \overline{\mathbf{v}}+\left|h_{B w}\right|^{2} \leq \overline{\mathbf{v}}^{H} \mathbf{Q}_{E} \overline{\mathbf{v}}+\left|h_{E w}\right|^{2} \\
& \left|\bar{v}_{n}\right|=1, \forall n=1, \ldots, N
\end{aligned}
$$

Define $\mathbf{V}=\overline{\mathbf{v v}}^{H}, \mathbf{V} \succeq 0$, and $\operatorname{rank}(\mathbf{V})=1$. Then, problem (P6) becomes to 
(P7): $\max _{\mathbf{V}} \operatorname{Tr}\left(\mathbf{Q}_{B} \mathbf{V}\right)$

$$
\begin{array}{ll}
\text { s.t. } & \operatorname{Tr}\left(\mathbf{Q}_{B} \mathbf{V}\right)-\operatorname{Tr}\left(\mathbf{Q}_{E} \mathbf{V}\right)+\left|h_{B w}\right|^{2}-\left|h_{E w}\right|^{2} \leq 0 \\
& \mathbf{V}_{n, n}=1, \forall n=1, \ldots, N+1 \\
& \mathbf{V} \succeq 0
\end{array}
$$

The problem (P7) is a standard convex semidefinite program (SDP) and can be optimally solved by the numerical solver CVX [19]. Generally, $\operatorname{rank}(\mathbf{V})=1$ is not satisfied. The Gaussian randomization method can derive a rank-one solution from the optimal solution to problem (P6). The optimal solution $\mathbf{V}$ to the problem (P7) is eigenvalue decomposed as $\mathbf{V}=\mathbf{U} \boldsymbol{\Sigma} \mathbf{U}^{H}$, where $\boldsymbol{\Sigma}=\operatorname{diag}\left(\lambda_{1}, \ldots \lambda_{N+1}\right)$ is a diagonal matrix and $\mathbf{U}=\left[\mathbf{e}_{1}, \ldots, \mathbf{e}_{N+1}\right]$ is a unitary matrix. A sub-optimal solution to (P7) is constructed as $\overline{\mathbf{v}}=\mathbf{U} \Sigma^{1 / 2} \mathbf{r}$ where $\quad \mathbf{r} \in \mathbb{C}^{N+1}$ is Gaussian variable satisfying $\mathcal{C N}\left(\mathbf{0}, \mathbf{I}_{N+1}\right)$ with zero meaning and covariance matrix $\mathbf{I}_{N+1}$. The objective value of (P6) is approximated as the closest to the optimal one attained by the best $\overline{\mathbf{v}}$ among all $\mathbf{r}^{\prime} s$ with independently generated Gaussian random vectors. Accordingly, the sub-optimal solution to the problem (P2) for any fixed beamforming vectors is derived by $\boldsymbol{\theta}=e^{j \arg \left(\overline{\mathbf{v}} \overline{\mathbf{v}}_{N+1}\right)_{[:: N]}}$.

In the next steps, we solve the optimization problem (P2) over the active beamforming vector $\mathbf{W}$, for any given $\boldsymbol{\Phi}$. Define $\quad \boldsymbol{\varphi}_{1}{ }^{H}=\mathbf{h}_{I B}^{H} \mathbf{\Phi} \mathbf{G}+\mathbf{h}_{A B}^{H} \quad, \quad \boldsymbol{\varphi}_{2}{ }^{H}=\mathbf{h}_{I E}^{H} \mathbf{\Phi} \mathbf{G}+\mathbf{h}_{A E}^{H} \quad, \quad$ the problem (P3) is equivalent to

$$
\begin{array}{ll}
\text { (P8): } \max _{\mathbf{w}} & \left|\boldsymbol{\varphi}_{1}{ }^{H} \mathbf{w}\right|^{2} \\
\text { s.t. } & \left|\boldsymbol{\varphi}_{1}{ }^{H} \mathbf{w}\right|^{2}-\left|\boldsymbol{\varphi}_{2}{ }^{H} \mathbf{w}\right|^{2} \leq 0 \\
& \|\mathbf{w}\|^{2} \leq P
\end{array}
$$

$$
\begin{aligned}
& \text { Let } \mathbf{Q}_{\mathbf{1}}=\boldsymbol{\varphi}_{1} \boldsymbol{\varphi}_{1}^{H}, \mathbf{Q}_{2}= \boldsymbol{\varphi}_{2} \boldsymbol{\varphi}_{2}^{H}, \mathbf{W}=\mathbf{w} \mathbf{w}^{H}, \text { we have } \\
& \text { (P9): } \quad \max _{\mathbf{W}} \operatorname{Tr}\left(\mathbf{Q}_{1} \mathbf{W}\right) \\
& \text { s.t. } \quad \operatorname{Tr}\left(\mathbf{Q}_{1} \mathbf{W}\right)-\operatorname{Tr}\left(\mathbf{Q}_{2} \mathbf{W}\right) \leq 0 \\
& \quad \operatorname{Tr}(\mathbf{W}) \leq P \\
& \mathbf{W} \succeq 0
\end{aligned}
$$

The rank-one constraints $\operatorname{Rank}(\mathbf{W})=1$ are omitted. However, as proved in [18], $\operatorname{Rank}(\mathbf{W})=1$ is always holding at the optimum of problem (P9). The proof is omitted here for brevity. The problem (P9) is a semi-definite program and can be solved by the numerical solver CVX [19].

Finally, with the solutions to problems (P7) and (P9), problem (P2) for each fixed value can be solved via alternating optimization, in which problems (P7) and (P9) are solved iteratively until the convergence. The $\mathrm{AO}$ algorithm is summarized in Table 1.

Table 1. Alternating Optimization.

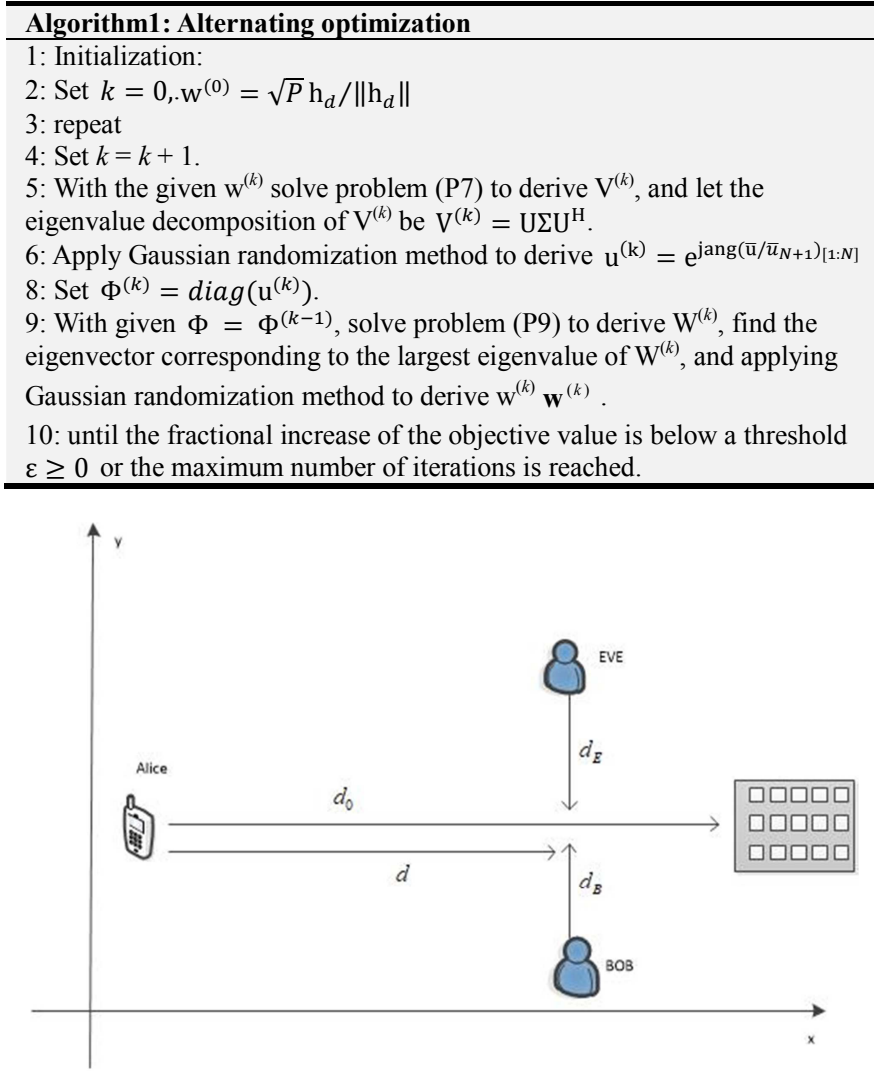

Figure 2. System simulation coordinate system.

\section{Simulation Results}

We consider an Equal-Height system supposing AP and users are consistent in height. As shown in Figure 3, the IRS with $N=N_{\mathrm{x}} \times N_{\mathrm{y}}$ reflecting elements total, $N_{\mathrm{x}}$ and $N_{\mathrm{y}}$ denote the numbers of reflecting elements along the $\mathrm{x}$-axis and y-axis.

The AP-IRS channel is dominated by the LoS link since the IRS of a surveillance system usually knows the AP's location to exploit LoS channel. We assume the Rician fading channel model for all channels because of small-scale fading. The path loss model is given by $L(d)=C_{0}\left(\frac{d}{D}\right)^{-\alpha}$, where $d$ is the distance of the link, $\alpha$ is the path loss exponent and $C_{0}$ is the signal attenuation at a reference distance $D=1$ meter. Then, the AP-IRS channel $\mathbf{G}$ is given by: $\mathbf{G}=\sqrt{\frac{\beta_{A I}}{1+\beta_{A I}}} \mathbf{G}^{L o S}+\sqrt{\frac{1}{1+\beta_{A I}}} \mathbf{G}^{N L O S}$, where $\beta_{A I}$ represent the Rician factor, $\mathbf{G}^{\text {LoS }}$ and $\mathbf{G}^{\text {NLoS }}$ are the deterministic LoS and Rayleigh fading components. The elements in $\mathbf{G}$ are then multiplied by the square root of the distance-dependent path loss with the path loss exponent denoted by $\alpha_{A I}$. The path loss exponents and Rician factors are set as $\alpha_{A I}=2$, $\alpha_{A B}=\alpha_{A E}=3.5, \alpha_{I B}=\alpha_{I E}=2.5, \beta_{A I}=\infty$, other links $\beta=0$. The number of Gaussian randomizations of the beamforming 
vectors is set to be 1000 . Other simulation parameters are set as: $\quad C_{0}=-30 \mathrm{~dB}, \quad P=0.1, \sigma_{B}^{2}=\sigma_{E}^{2}=-80 \mathrm{dBm}$, $\mathrm{d}_{B}=2 m, d_{E}=3 m, N=50$ (if not specified otherwise). The AP-user and IRS-user link distances are given by $\mathrm{d}_{1}=\sqrt{d^{2}+d_{B}^{2}} \quad, \quad \mathrm{~d}_{2}=\sqrt{d^{2}+d_{E}^{2}} \quad, \quad \mathrm{~d}_{3}=\sqrt{\left(d_{0}-d\right)^{2}+d_{B}^{2}}$, $\mathrm{d}_{4}=\sqrt{\left(d_{0}-d\right)^{2}+d_{E}^{2}}$.

\subsection{Monitor-unaware Surveillance}

We consider the setup where B and E are $35 \mathrm{~m}$ away from $\mathrm{AP}$, the position of the AP is fixed while the distance between IRS and AP is increasing increases from 20 to 70 . As shown in Figure 3, the closer the IRS is to the supervisor, the higher the accessibility rate of the supervisor. When the IRS location exceeds the distance range, the IRS can only improve the accessibility rate of supervisors little. And as we can see from the figure, the right IRS location can increase the accessibility rate of both supervisor and suspects. As shown in the figure, the supervisor B cannot monitor the information of $\mathrm{E}$ without the assistance of IRS, since the achievable rate of $E$ is always lower than that of B.

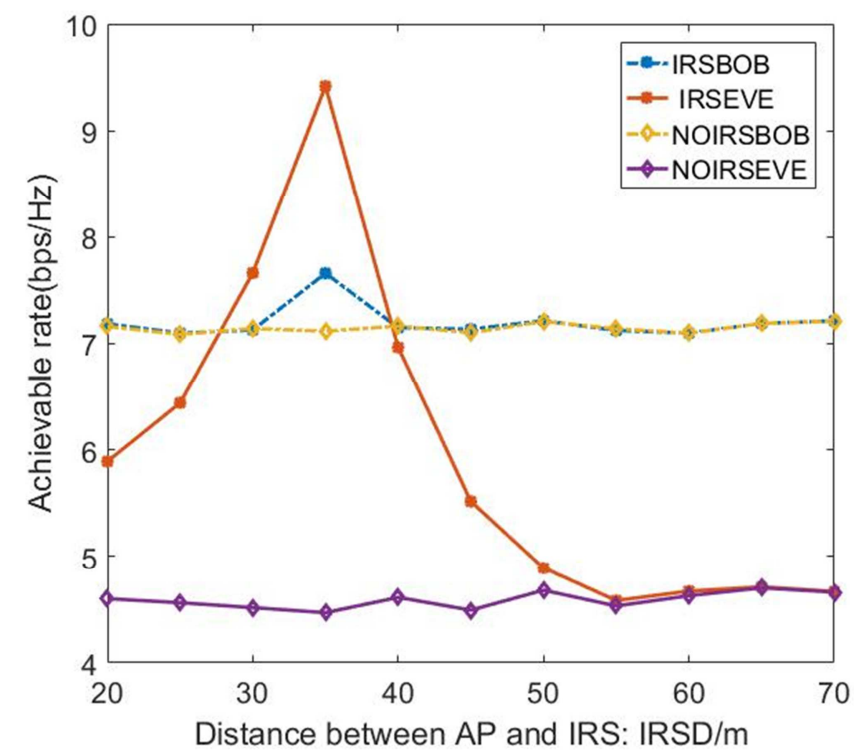

Figure 3. Achievable rate vs. the distance between AP and IRS.

As shown in Figure 4, we compare the achievable rate of the IRS-assisted system and system without IRS with different distances between AP and B. We set the distance $d_{0}=35 \mathrm{~m}$ between AP and IRS, while B moves along the $\mathrm{X}$-axis. First, for the scheme with IRS, it is evident that the achievable rate of $E$ is dramatically improved. On the premise that $B$ is not disturbed, $\mathrm{E}$ can monitor the information transmitted from $\mathrm{AP}$ to $\mathrm{B}$. Moreover, as the distance between AP and B increases, the achievable rate of $\mathrm{B}$ keeps decreasing, and $\mathrm{E}$ can always maintain a high achievable rate. Again, it can be shown from Figure 5 that the monitor $\mathrm{E}$ cannot decode the information of the suspicious link from AP to B without the assistance of the IRS.

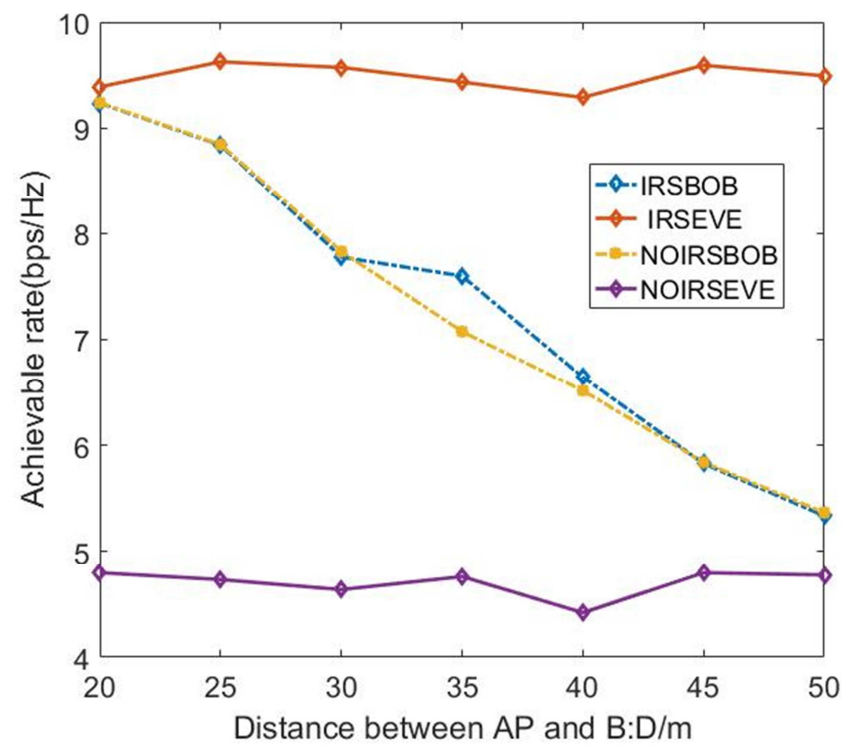

Figure 4. Achievable rate vs. the distance between $A P$ and $B$.

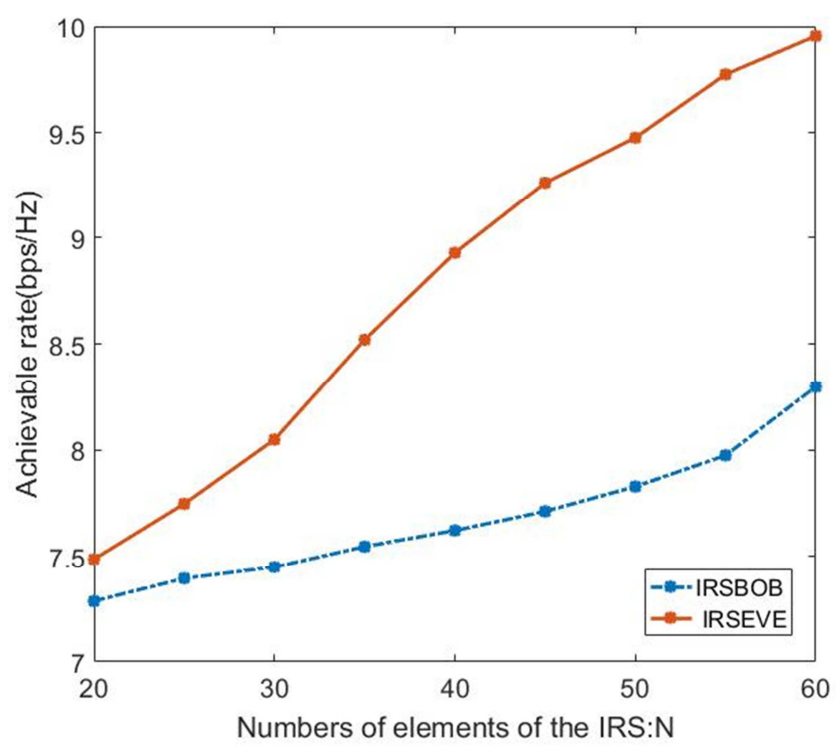

Figure 5. Achievable rate vs. the number of the elements of the IRS.

Figure 5 shows the achievable rate with different number of IRS elements. Both the achievable rate of the monitor $\mathrm{E}$ and the suspicious receiver $B$ increase as the number of the elements of the IRS increases. It is necessary to point out that the achievable rate of $E$ increases with a faster speed than that of B. Such a performance gain is attributed to two aspects. 1) the achievable rate increases greatly because of the increase of the number of reflective elements; 2) monitor can obtain a higher achievable rate by optimizing the reflection coefficient matrix.

\subsection{Monitor-aware Surveillance}

In Figure 6, we compare the achievable rates of $\mathrm{B}$ and $\mathrm{E}$ with or without the assistance of the IRS. The receivers B and E are 35 meters away from the AP. We assume the IRS moves along the $\mathrm{X}$ axis. The achievable rate by jointly optimizing the beamforming at the AP and reflecting matrix at the IRS in the 
monitor-aware case is less sensitive to the location of the IRS compared to that in the monitor-unaware case. Within a certain range of the distance between IRS and AP, the achievable rate of the monitor changes little. However, the achievable decreases when the IRS is near to the receivers.

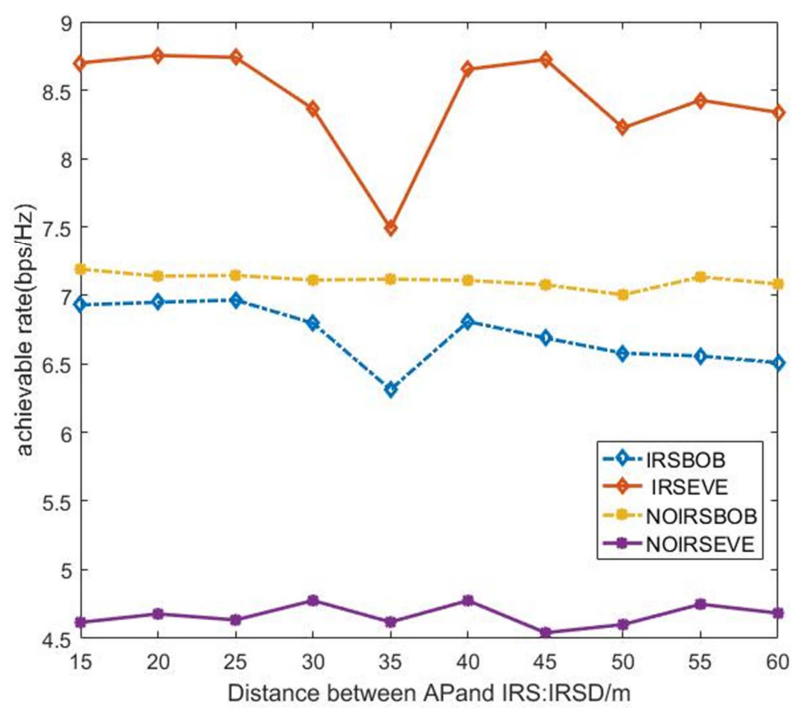

Figure 6. Achievable rate vs. the distance between AP and IRS.

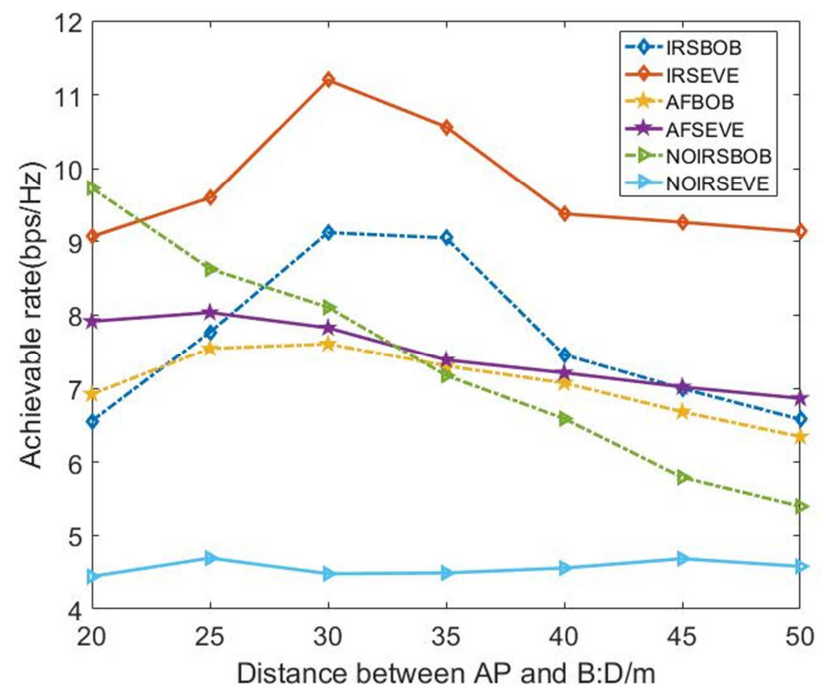

Figure 7. The relationship between achievable rate with B's location.

In Figure 7 we compare the achievable rate of different schemes, namely the IRS-assistance, no IRS case and relay-assistance scheme assuming the transmit power in each scheme is the same. In the relay-assistance scheme, the relay forwards the signals with AF relaying. We consider the setup where the IRS and E are 35 meters away from the AP and B moves along the $\mathrm{X}$-axis. For the system without the IRS, we can observe that the monitor suffers more SNR loss due to signal attenuation. However, deploying an IRS improves the monitor's achievable rate significantly. And, a larger AP-monitor distance leads to a high monitor achievable rate in IRS-assisted surveillance systems. It's clear in Figure 7 that the performance of the monitor-aware surveillance system is greatly improved through jointly optimization. Supervisor can get a larger achievable rate than surveillance system without the assistance of IRS. Compared with the relay-assisted surveillance system, E achieves larger achievable rate in IRSassisted surveillance system. Therefore, to design a monitor-aware surveillance system, it is more energy-saving to use IRS than using relaying.

\section{Conclusion}

In this paper, we designed an IRS-assisted surveillance wireless communication system to improve the achievable rate. Specifically, active beamforming and passive beamforming are optimized jointly to maximize the achievable rate of IRS-assisted surveillance systems by SDR and alternating optimization techniques. Simulation results demonstrate the achievable rate improvement signal coverage extension achieved by deploying the IRS as compared to the conventional setup with relays or without IRS. We can also improve the surveillance systems by adjusting the position of the reflective surface and the number of reflective surfaces. The research of joint optimization of wireless supervision system and IRS is in the initial stage, there are still many problems worth study. For example, due to the location of the monitored uncertainty, the best intelligent reflector selection in intelligent reflector group and multi-reflector joint supervision optimization problem; In the case of multi-intelligent reflector surveillance systems, the robustness of surveillance systems is also a problem worth pondering.

\section{Acknowledgements}

This research was supported in part by the Fundamental Research Funds for the Central Universities in China (No. 2019B22614).

\section{References}

[1] N. Kshetri. 5G in E-Commerce Activities. IT Prof., 2018, 4, pp. 73-77.

[2] J. L. Massey, "An introduction to contemporary cryptology," Proc. IEEE, vol. 76, no. 5, pp. 533-549, May 1988.

[3] W. Lu, K. An, T. Liang, Robust beamforming design for sum secrecy rate maximization in multibeam satellite systems. IEEE Transactions on Aerospace and Electronic Systems, 2019, 55 (3): $1568-1572$.

[4] Zou Y, Wang X, Shen W. Optimal relay selection for physical-layer security in cooperative wireless networks. IEEE journal on selected areas in communications, 2013, vol. 31, no. 10, 2099-2111.

[5] W. Wang, K. C. Teh, K H. Li, Artificial noise aided physical layer security in multi-antenna small-cell networks. IEEE Transactions on Information Forensics and Security, 2017, 12 (6), pp. 1470-1482.

[6] F. Oggier and B. Hassibi, The secrecy capacity of the MIMO wiretap channel, IEEE Trans. Inf. Theory, 2011, vol. 57, no. 8, pp. 4961-4972. 
[7] J. Zhu, Y. Zou, G. Wang, Y. Yao and G. K. Karagiannidis, On secrecy performance of antenna-selection-aided MIMO systems against eavesdropping, IEEE Transactions on Vehicular Technology, 2016, vol. 65, no. 1, pp. 214-225, Jan. 2016, doi: 10.1109/TVT.2015.2397195.

[8] B. V. Nguyen and K. Kim, Secrecy outage probability of optimal relay selection for secure AF cooperative networks, IEEE Communications Letters, Dec. 2015, vol. 19, no. 12, pp. 2086-2089, doi: 10.1109/LCOMM.2015.2486768.

[9] Q. Wu, R. Zhang. Towards smart and reconfigurable environment: Intelligent reflecting surface aided wireless network. IEEE Communications Magazine, 2019, vol. 58, no. 1, pp. 106-112.

[10] Q. Wu, R. Zhang. Intelligent reflecting surface enhanced wireless network: Joint active and passive beamforming design $[\mathrm{C}] / / 2018$ IEEE Global Communications Conference (GLOBECOM). IEEE, 2018: 1-6.

[11] W. Chen, X. Ma, Z. Li, et al. Sum-rate maximization for intelligent reflecting surface based terahertz communication systems, 2019 IEEE/CIC International Conference on Communications Workshops in China (ICCC Workshops). IEEE, 2019: 153-157.

[12] M. Cui, G. Zhang, R. Zhang. Secure wireless communication via intelligent reflecting surface. IEEE Wireless Communications Letters, 2019, vol. 8, no. 5, pp. 1410-1414.
[13] X. Yu, D. Xu, R. Schober. Enabling secure wireless communications via intelligent reflecting surfaces. 2019 IEEE Global Communications Conference (GLOBECOM). IEEE, 2020 .

[14] J. Xu, L. Duan, R. Zhang. Proactive eavesdropping via jamming for rate maximization over Rayleigh fading channels. IEEE Wireless Communications Letters, 2015, vol. 5, no. 1, pp. 80-83.

[15] X. Guan, Q. Wu, R. Zhang. Intelligent reflecting surface assisted secrecy communication via joint beamforming and jamming, arXiv preprint arXiv: 1907. 12839, 2019.

[16] Y. Zeng and R. Zhang, "Active eavesdropping via spoofing relay attack,"in Proc. Int. Conf. Acoust., Speech, Signal Process., Shanghai, Mar. 2016, pp. 2159-2163.

[17] Y. Zeng and R. Zhang, Wireless information surveillance via proactive eavesdropping with spoofing relay, IEEE Journal of Selected Topics in Signal Processing, 2016, vol. 10, no. 8, pp. 1449-1461.

[18] Z. Q. Luo, W. K. Ma, A. M. C. So, et al. "Semidefinite relaxation of quadratic optimization problems," IEEE Signal Processing Magazine, 2010, vol. 27, no. 3, pp. 20-34.

[19] M. Grant and S. Boyd. "CVX: Matlab software for disciplined convex programming,” Apr. 2011 [Online]. Available: http://cvxr.com/cvx/. 